Supplement of Biogeosciences, 12, 4841-4860, 2015

http://www.biogeosciences.net/12/4841/2015/

doi:10.5194/bg-12-4841-2015-supplement

(C) Author(s) 2015. CC Attribution 3.0 License.

(c) (i)

Supplement of

\title{
Multi-molecular tracers of terrestrial carbon transfer across the pan-Arctic: comparison of hydrolyzable components with plant wax lipids and lignin phenols
}

\section{Feng et al.}

Correspondence to: X. Feng (xfeng@ibcas.ac.cn)

The copyright of individual parts of the supplement might differ from the CC-BY 3.0 licence. 
Table S1: Abundances of solvent-extractable $\boldsymbol{n}$-alkanes, FAs, and hydrolysable compounds in pan-arctic river sediments (mg/g OC). Analytical error associated with biomarker analysis is typically $<10 \%$. The $n$-alkane and FA data are from Vonk et al., 2008 (Kalix), van Dongen et al., 2008a (GRARs), and Drenzek et al., 2007 (Mackenzie).

\begin{tabular}{|c|c|c|c|c|c|c|c|c|c|c|}
\hline & Kalix & $\mathbf{O b}^{\prime}$ & Yenisey & Lena & Indigirka & Kolyma & Colville1 & Colville2 & Yukon & Mackenzie \\
\hline \multicolumn{11}{|c|}{ SOLVENT-EXTRACTABLE LIPIDS } \\
\hline \multicolumn{11}{|l|}{$n$-Alkanes } \\
\hline $\mathrm{C}_{20}$ & 0.01 & 0.02 & 0.01 & 0.01 & 0.01 & 0.01 & 0.01 & 0.02 & & 0.03 \\
\hline $\mathrm{C}_{21}$ & 0.02 & 0.07 & 0.03 & 0.03 & 0.07 & 0.05 & 0.02 & 0.05 & 0.02 & 0.04 \\
\hline $\mathrm{C}_{22}$ & 0.01 & 0.07 & 0.03 & 0.02 & 0.05 & 0.03 & 0.02 & 0.04 & 0.01 & 0.03 \\
\hline $\mathrm{C}_{23}$ & 0.05 & 0.21 & 0.09 & 0.05 & 0.14 & 0.11 & 0.06 & 0.14 & 0.04 & 0.04 \\
\hline $\mathrm{C}_{24}$ & 0.02 & 0.11 & 0.03 & 0.02 & 0.06 & 0.04 & 0.02 & 0.04 & 0.02 & 0.03 \\
\hline $\mathrm{C}_{25}$ & 0.07 & 0.26 & 0.11 & 0.06 & 0.19 & 0.14 & 0.06 & 0.12 & 0.08 & 0.04 \\
\hline$C_{26}$ & 0.02 & 0.12 & 0.03 & 0.02 & 0.05 & 0.03 & 0.02 & 0.03 & 0.02 & 0.03 \\
\hline$C_{27}$ & 0.08 & 0.30 & 0.18 & 0.10 & 0.34 & 0.28 & 0.10 & 0.16 & 0.19 & 0.06 \\
\hline $\mathrm{C}_{28}$ & 0.01 & 0.08 & 0.02 & 0.02 & 0.05 & 0.03 & 0.01 & 0.02 & 0.02 & 0.02 \\
\hline $\mathrm{C}_{29}$ & 0.04 & 0.24 & 0.15 & 0.09 & 0.30 & 0.20 & 0.07 & 0.10 & 0.11 & 0.06 \\
\hline$C_{30}$ & 0.01 & 0.05 & 0.02 & 0.01 & 0.04 & 0.02 & 0.01 & 0.02 & 0.00 & 0.02 \\
\hline$C_{31}$ & 0.04 & 0.21 & 0.16 & 0.09 & 0.26 & 0.17 & 0.06 & 0.07 & 0.08 & 0.05 \\
\hline$C_{32}$ & 0.00 & 0.03 & 0.01 & 0.01 & 0.03 & 0.01 & 0.01 & 0.02 & 0.02 & 0.01 \\
\hline $\mathrm{C}_{33}$ & 0.00 & 0.04 & 0.04 & 0.02 & 0.08 & 0.04 & 0.02 & 0.03 & 0.03 & 0.02 \\
\hline$C_{34}$ & & 0.00 & 0.00 & 0.01 & 0.02 & 0.00 & 0.00 & 0.01 & 0.01 & 0.01 \\
\hline HMW $n$-alkanes $\left(C_{20}-C_{34}\right)$ & 0.38 & 1.83 & 0.91 & 0.55 & 1.70 & 1.16 & 0.49 & 0.87 & 0.65 & 0.50 \\
\hline FAs & & & & & & & & & & \\
\hline
\end{tabular}




\begin{tabular}{|c|c|c|c|c|c|c|c|c|c|c|}
\hline $\mathrm{C}_{16}$ & 0.09 & & 0.02 & & & & 0.08 & 0.07 & 0.15 & 2.37 \\
\hline $\mathrm{C}_{18}$ & 0.03 & & 0.02 & & & & 0.03 & 0.03 & 0.05 & 0.14 \\
\hline$C_{20}$ & 0.04 & 0.01 & 0.00 & 0.02 & 0.02 & 0.04 & 0.04 & 0.04 & 0.08 & 0.04 \\
\hline$C_{21}$ & 0.02 & 0.01 & 0.00 & 0.01 & 0.01 & 0.02 & 0.01 & 0.01 & 0.02 & 0.02 \\
\hline$C_{22}$ & 0.15 & 0.03 & 0.02 & 0.05 & 0.08 & 0.19 & 0.08 & 0.08 & 0.19 & 0.08 \\
\hline$C_{23}$ & 0.04 & 0.01 & 0.01 & 0.02 & 0.03 & 0.08 & 0.03 & 0.03 & 0.07 & 0.04 \\
\hline$C_{24}$ & 0.16 & 0.05 & 0.05 & 0.07 & 0.12 & 0.29 & 0.17 & 0.18 & 0.29 & 0.14 \\
\hline $\mathrm{C}_{25}$ & 0.03 & 0.02 & 0.01 & 0.02 & 0.03 & 0.05 & 0.03 & 0.03 & 0.06 & 0.03 \\
\hline$C_{26}$ & 0.08 & 0.05 & 0.05 & 0.05 & 0.10 & 0.15 & 0.13 & 0.14 & 0.21 & 0.07 \\
\hline $\mathrm{C}_{27}$ & 0.01 & 0.01 & 0.01 & 0.01 & 0.02 & 0.02 & 0.02 & 0.02 & 0.03 & 0.02 \\
\hline $\mathrm{C}_{28}$ & 0.03 & 0.04 & 0.04 & 0.03 & 0.08 & 0.08 & 0.10 & 0.10 & 0.16 & 0.03 \\
\hline $\mathrm{C}_{29}$ & 0.00 & 0.00 & 0.01 & 0.00 & 0.01 & 0.01 & 0.01 & 0.01 & 0.02 & 0.00 \\
\hline$C_{30}$ & 0.01 & 0.02 & 0.02 & 0.02 & 0.04 & 0.03 & 0.04 & 0.04 & 0.07 & 0.01 \\
\hline HMW FAs $\left(C_{20}-C_{30}\right)$ & 0.57 & 0.26 & 0.22 & 0.31 & 0.55 & 0.96 & 0.67 & 0.68 & 1.19 & 0.48 \\
\hline \multicolumn{11}{|c|}{ HYDROLYSABLE COMPOUNDS } \\
\hline \multicolumn{11}{|l|}{ b-FAs } \\
\hline$i-C_{14}$ & 0.09 & 0.11 & 0.13 & 0.04 & 0.06 & 0.07 & 0.06 & 0.05 & 0.06 & \\
\hline $\mathrm{C}_{14}$ & 0.03 & 0.21 & 0.20 & 0.10 & 0.14 & 0.00 & 0.27 & 0.21 & 0.17 & \\
\hline$i-C_{15}$ & 0.17 & 0.11 & 0.13 & 0.08 & 0.09 & 0.13 & 0.17 & 0.13 & 0.12 & \\
\hline$a-C_{15}$ & 0.18 & 0.17 & 0.18 & 0.09 & 0.10 & 0.14 & 0.17 & 0.14 & 0.15 & \\
\hline$C_{15}$ & 0.06 & 0.09 & 0.07 & 0.04 & 0.06 & 0.09 & 0.07 & 0.06 & 0.06 & \\
\hline$i-C_{16}$ & 0.07 & 0.06 & 0.07 & 0.03 & 0.06 & 0.06 & 0.07 & 0.06 & 0.06 & \\
\hline$C_{16: 1}$ & 0.15 & 0.16 & 0.16 & 0.06 & 0.09 & 0.06 & 0.41 & 0.26 & 0.17 & \\
\hline$C_{16}$ & 0.77 & 0.93 & 0.72 & 0.19 & 0.55 & 0.90 & 0.68 & 0.76 & 0.59 & \\
\hline$i-C_{17}$ & 0.04 & 0.00 & 0.02 & 0.00 & 0.04 & 0.00 & 0.05 & 0.05 & 0.01 & \\
\hline
\end{tabular}




\begin{tabular}{|c|c|c|c|c|c|c|c|c|c|}
\hline$a-C_{17}$ & 0.06 & 0.04 & 0.04 & 0.01 & 0.00 & 0.05 & 0.03 & 0.03 & 0.05 \\
\hline$C_{17}$ & 0.06 & 0.05 & 0.00 & 0.00 & 0.00 & 0.15 & 0.03 & 0.04 & 0.04 \\
\hline$C_{18: 1}$ & 0.45 & 0.48 & 0.65 & 0.13 & 0.41 & 0.74 & 0.36 & 0.25 & 0.17 \\
\hline $\mathrm{C}_{18}$ & 0.25 & 0.27 & 0.19 & 0.07 & 0.14 & 0.21 & 0.20 & 0.22 & 0.13 \\
\hline $\mathrm{C}_{19}$ & 0.02 & 0.01 & 0.02 & 0.00 & 0.01 & 0.01 & 0.03 & 0.10 & 0.01 \\
\hline$C_{20}$ & 0.12 & 0.05 & 0.04 & 0.01 & 0.06 & 0.05 & 0.19 & 0.15 & 0.09 \\
\hline$C_{21}$ & 0.03 & 0.01 & 0.01 & 0.01 & 0.01 & 0.01 & 0.06 & 0.05 & 0.01 \\
\hline$C_{22}$ & 0.14 & 0.03 & 0.02 & 0.02 & 0.02 & 0.02 & 0.18 & 0.23 & 0.08 \\
\hline $\mathrm{C}_{23}$ & 0.02 & 0.01 & 0.01 & 0.01 & 0.01 & 0.01 & 0.06 & 0.11 & 0.02 \\
\hline $\mathrm{C}_{24}$ & 0.09 & 0.04 & 0.01 & 0.02 & 0.02 & 0.01 & 0.13 & 0.35 & 0.04 \\
\hline $\mathrm{C}_{25}$ & 0.01 & 0.01 & 0.00 & 0.00 & 0.02 & 0.00 & 0.03 & 0.06 & 0.01 \\
\hline$C_{26}$ & 0.05 & 0.04 & 0.01 & 0.01 & 0.07 & 0.01 & 0.03 & 0.14 & 0.01 \\
\hline$C_{27}$ & 0.01 & 0.01 & 0.00 & 0.00 & 0.01 & 0.00 & 0.01 & 0.02 & 0.00 \\
\hline $\mathrm{C}_{28}$ & 0.03 & 0.03 & 0.01 & 0.00 & 0.05 & 0.02 & 0.01 & 0.05 & 0.00 \\
\hline $\mathrm{C}_{29}$ & 0.00 & 0.01 & 0.00 & 0.00 & 0.00 & 0.00 & 0.00 & 0.00 & 0.00 \\
\hline $\mathrm{C}_{30}$ & 0.01 & 0.02 & 0.00 & 0.00 & 0.00 & 0.02 & 0.01 & 0.01 & 0.00 \\
\hline LMW b-FAs $\left(C_{14}-C_{19}\right)$ & 2.41 & 2.70 & 2.57 & 0.85 & 1.75 & 2.61 & 2.59 & 2.36 & 1.79 \\
\hline HMW b-FAs $\left(C_{20}-C_{30}\right)$ & 0.51 & 0.26 & 0.12 & 0.08 & 0.27 & 0.15 & 0.71 & 1.17 & 0.26 \\
\hline \multicolumn{10}{|l|}{$\omega$-Hydroxy FAs } \\
\hline $\mathrm{C}_{16}$ & 0.21 & 0.44 & 0.49 & 0.23 & 0.82 & 1.52 & 0.70 & 0.67 & 1.19 \\
\hline $\mathrm{C}_{18: 1}$ & 0.00 & 0.14 & 0.09 & 0.07 & 0.34 & 0.46 & 0.31 & 0.38 & 1.34 \\
\hline $\mathrm{C}_{18}$ & 0.12 & 0.16 & 0.16 & 0.07 & 0.44 & 0.71 & 0.34 & 0.29 & 0.54 \\
\hline $\mathrm{C}_{20}$ & 0.30 & 0.49 & 0.42 & 0.22 & 0.93 & 1.69 & 0.79 & 0.82 & 1.46 \\
\hline$C_{22}$ & 0.50 & 0.99 & 0.76 & 0.40 & 1.54 & 3.13 & 1.53 & 1.61 & 2.42 \\
\hline $\mathrm{C}_{24}$ & 0.24 & 0.63 & 0.34 & 0.14 & 0.51 & 1.08 & 0.83 & 0.92 & 0.24 \\
\hline $\mathrm{C}_{26}$ & 0.05 & 0.19 & 0.12 & 0.03 & 0.20 & 0.20 & 0.28 & 0.11 & 0.05 \\
\hline
\end{tabular}




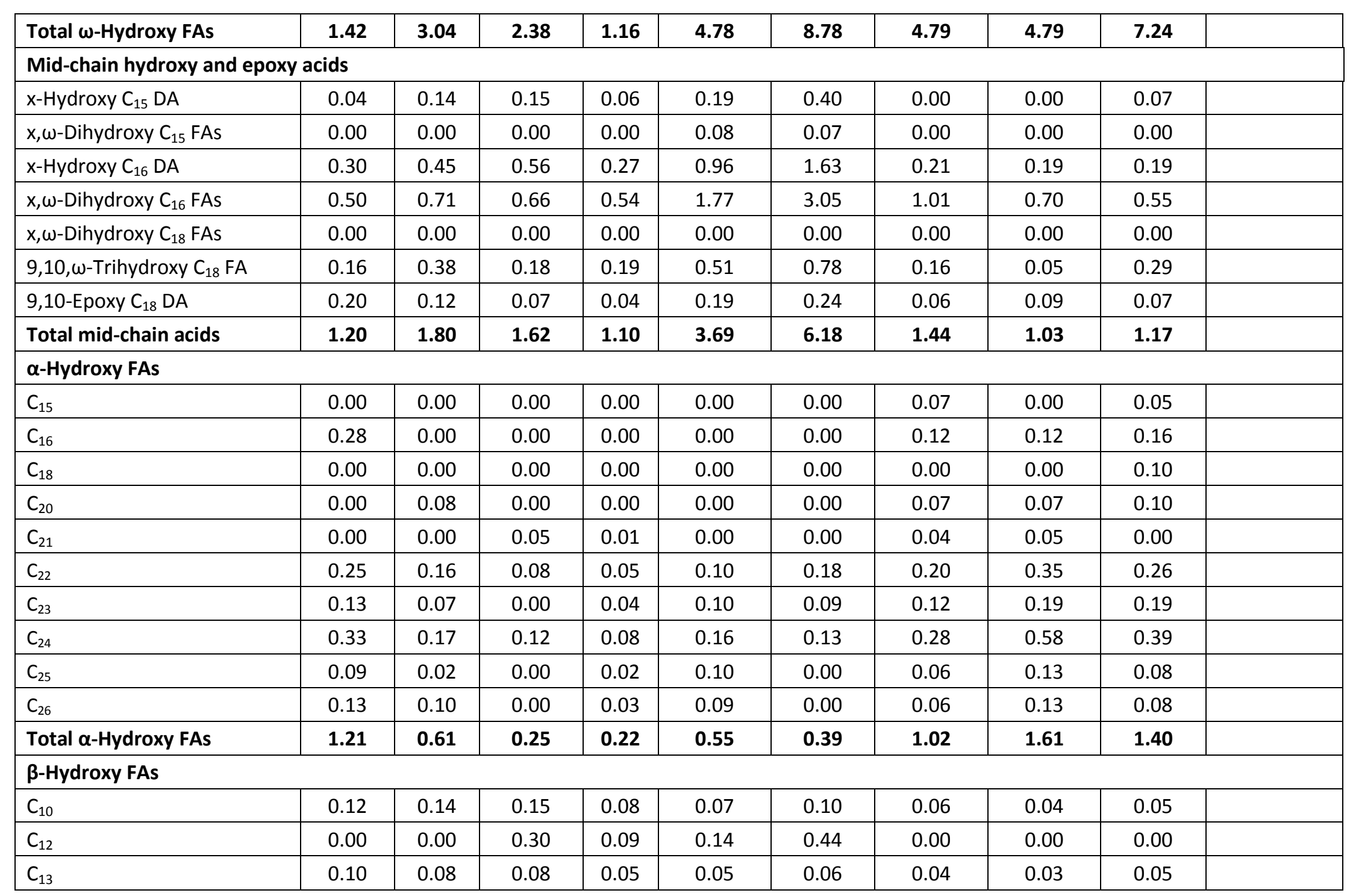




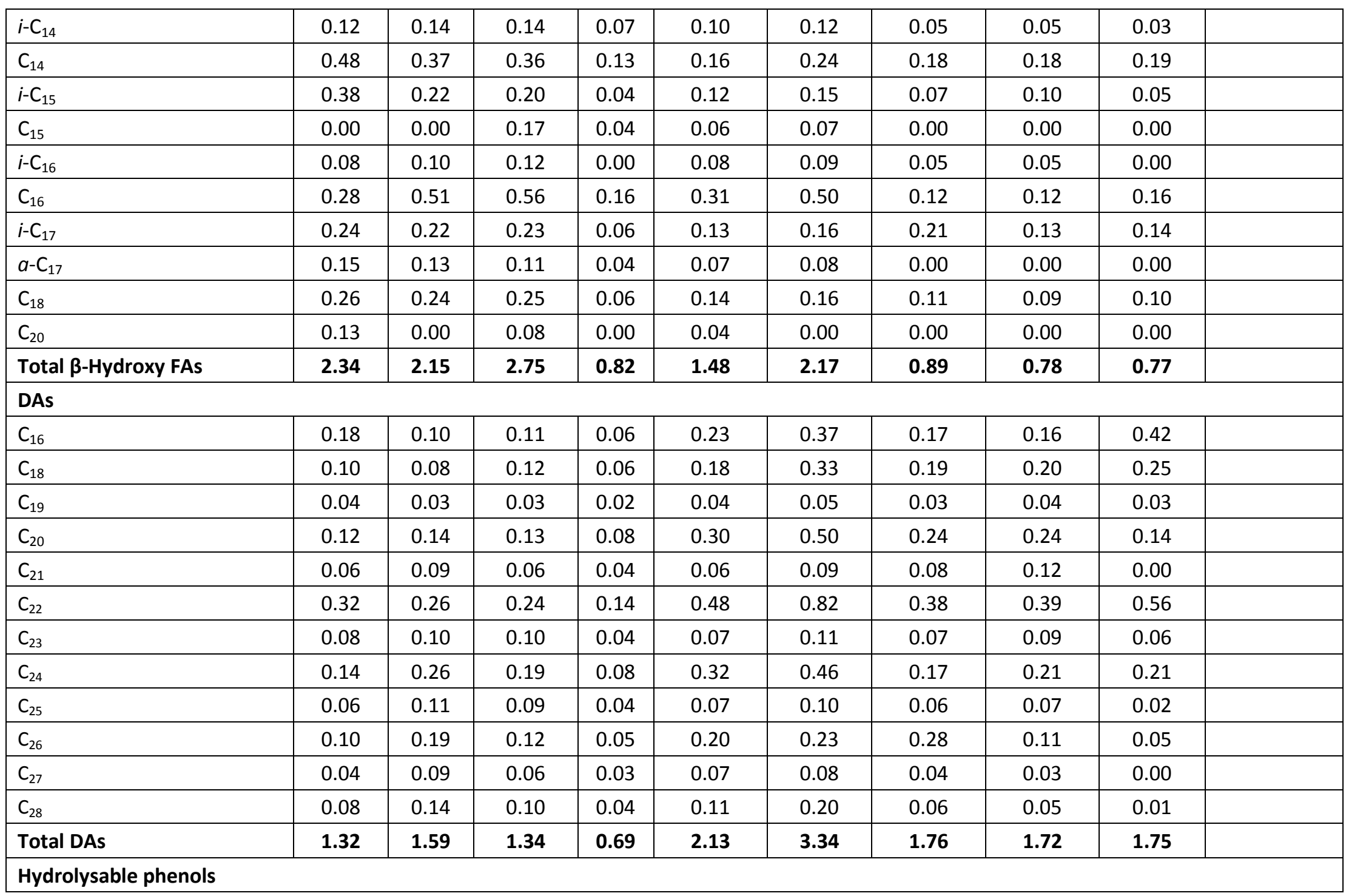




\begin{tabular}{|c|c|c|c|c|c|c|c|c|c|}
\hline Vanillin & 0.05 & 0.03 & 0.02 & 0.04 & 0.05 & 0.50 & 0.00 & 0.00 & 0.03 \\
\hline Acetovanillone & 0.02 & 0.03 & 0.02 & 0.03 & 0.04 & 0.07 & 0.00 & 0.00 & 0.04 \\
\hline Vanillic acid & 0.04 & 0.06 & 0.06 & 0.04 & 0.10 & 0.21 & 0.03 & 0.05 & 0.09 \\
\hline Syringaldehyde & 0.03 & 0.02 & 0.06 & 0.02 & 0.04 & 0.04 & 0.02 & 0.00 & 0.03 \\
\hline Syringic acid & 0.04 & 0.04 & 0.04 & 0.02 & 0.09 & 0.14 & 0.04 & 0.05 & 0.06 \\
\hline Ferulic acid & 0.04 & 0.09 & 0.05 & 0.04 & 0.23 & 0.48 & 0.10 & 0.16 & 0.11 \\
\hline Total hydrolysable phenols & 0.23 & 0.28 & 0.26 & 0.18 & 0.55 & 1.45 & 0.20 & 0.26 & 0.35 \\
\hline
\end{tabular}

